\title{
Efecto de la inoculación con rizobios y la fertilización fosfatada sobre la nodulación y producción de alfalfa (Medicago sativa L.) en el centro de Santa Fe (Argentina)
}

Toniutti, M. A. y Fornasero, L. V.

\begin{abstract}
RESUMEN
La simbiosis entre rizobios y alfalfa (Medicago sativa L.) contribuye a la incorporación de nitrógeno en los agroecosistemas a través de la fijación biológica, que dependerá de la adecuada provisión de nutrientes disponibles en el suelo. El objetivo de este trabajo fue evaluar el efecto de la inoculación con Ensifer meliloti y la fertilización con 0, 20 y $40 \mathrm{~kg} / \mathrm{ha}$ de fósforo sobre la nodulación y productividad de una pastura de alfalfa en el centro de la provincia de Santa Fe. La inoculación y la aplicación de dosis crecientes de fósforo aumentaron el número de nódulos, biomasa nodular y la producción del forraje de las plantas. La técnica de la inoculación condujo a un incremento de la producción de materia seca entre 23 y 58 \% para los tratamientos con el máximo nivel de fertilizante a los 120, 180 y 240 días de la siembra. La adición de la cepa de rizobio utilizada como inoculante y la fertilización fosfatada constituyeron una estrategia favorable para incrementar la producción de alfalfa.
\end{abstract}

Palabras clave: Ensifer meliloti; fósforo; producción de forraje.

Toniutti, M. A. and Fornasero, L. V., 2020. Effect of rhizobia inoculation and phosphate fertilization on the nodulation and production of alfalfa (Medicago sativa L.) In the center of Santa Fe (Argentina). Agriscientia 37 (2): $1-10$

\section{SUMMARY}

The symbiosis between rhizobia and alfalfa (Medicago sativa L.) contributes to the incorporation of nitrogen in agroecosystems through biological fixation, which will depend on the adequate supply of available nutrients in the soil. The objective of this work was to evaluate the effect of the inoculation with Ensifer meliloti and the fertilization with 0,20 and $40 \mathrm{~kg} / \mathrm{ha}$ of phosphorus on the nodulation and productivity of an alfalfa pasture in the center of Santa Fe province. The inoculation and the application of increasing doses of phosphorus 
boosted the number of nodules, nodular biomass and forage production of the plants. The technique of inoculation led to a rise in dry matter production between 23 and $58 \%$ with the highest level of fertilizer at 120, 180 and 240 days after planting. The addition of the strain of rhizobium selected as inoculant and phosphate fertilization were a favorable strategy to increase the production of alfalfa.

Key words: Ensifer meliloti; phosphorus; forage production.

Toniutti, M. A.1 (ORCID: 0000-0001-8168-1386) y Fornasero, L. V. (ORCID: 0000-0003-0313-2079): Facultad de Ciencias Agrarias, Universidad Nacional del Litoral. R. P. Kreder 2805, (3080 HOF), Esperanza, Santa Fe, Argentina. Correspondencia a: matoniut@fca.unl.edu.ar

\section{INTRODUCCIÓN}

La alfalfa (Medicago sativa L.) es la principal forrajera del país y la base de la producción de carne y leche en la Región Pampeana (Basigalup, Rossanigo y Ballario, 2007). Esta leguminosa presenta un elevado valor nutritivo, excelente palatabilidad y digestibilidad, comparada con otras especies. Además contribuye a la incorporación de nitrógeno $(\mathrm{N})$ en los agroecosistemas a través de la fijación biológica (Anglade, Billen y Garnier, 2015), con un consecuente beneficio tanto económico como ambiental (Campillo, Urquiaga, Pino y Montenegro, 2003; Jensen y Hauggaard-Nielsen, 2003), ya que permite reducir significativamente la aplicación de fertilizantes nitrogenados.

Las plantas de alfalfa presentan un contenido de proteínas elevado y por consiguiente, altos requerimientos nitrogenados (Howarth, 1988). Estos altos consumos pueden provocar una pérdida de la fertilidad nitrogenada del suelo o una restricción en el crecimiento, si no existe un sistema de reciclaje parcial del $\mathrm{N}$ unido a un eficiente sistema de fijación biológica de nitrógeno (FBN). Por lo tanto, la cualidad de la alfalfa de producir forraje y mantener o aumentar la fertilidad nitrogenada de los suelos, dependería en gran medida de su capacidad para incorporar $\mathrm{N}$ de la atmósfera por asociación con rizobios específicos (Racca et al., 2001). Esta simbiosis es capaz de establecerla con Ensifer meliloti o Ensifer medicae (Silva, Kan y Martínez-Romero, 2007) y con otros rizobios como las cepas de tipo Oregon, Rhizobium sp. Or191 inicialmente aislada en EE.UU. y Rhizobium sp. Cepa LPU83, en Argentina (Tejerizo et al., 2016).

En el país, el cultivo de alfalfa se realiza mayoritariamente bajo condiciones de secano con potenciales productivos, principalmente en la época primavero-estivo-otoñal, cercanos a $20 \mathrm{t}$ de materia seca/ha año (Basigalup et al., 2007). La variabilidad productiva de los alfalfares ha sido relacionada con las características y aptitudes de los suelos y con la dotación de nutrientes disponibles para el cultivo (Quiñonez, Romero, Dalla Fontana, Longoni y Colombo, 2008). El fósforo (P) es un nutriente crítico para maximizar la calidad y producción de materia seca de la alfalfa (Vivas y Guaita, 1997). Este nutriente interviene en múltiples procesos, tales como fotosíntesis, desarrollo de la biomasa aérea y radical y capacidad y actividad de los nódulos (Ribet y Drevon, 1995; Díaz Zorita y Gambaudo, 2007). La deficiencia de P en los suelos de la Región Pampeana húmeda constituye una de las principales limitantes para la producción de los cultivos (Sainz Rozas, Echeverría y Angelini, 2012) y se ha demostrado que esta situación restringe la producción forrajera de las pasturas de alfalfa (Berardo y Marino, 2000; Sevilla y Agnusdei, 2016). En Santa Fe, los suelos en condiciones prístinas presentaban niveles medios de $\mathrm{P}$ disponible (10$20 \mathrm{mg} / \mathrm{kg}$ ) en el este y niveles altos (>20 mg/kg) hacia el oeste de la provincia (Darwich, 1983). La continua remoción y escasa reposición del $\mathrm{P}$ extraído han resultado en la disminución del $\mathrm{P}$ disponible, y algunas áreas han pasado de rangos de valores promedios altos a medios o bajos (Sainz Rozas et al., 2012).

Para un ambiente determinado, después de la deficiencia hídrica, el $\mathrm{P}$ y el $\mathrm{N}$ son frecuentemente los factores que más condicionan la producción forrajera. El $P$ es necesario para lograr un establecimiento exitoso y un desarrollo profuso de las raíces. Además, en condiciones adversas como suelos fríos o sequías, que reducen la absorción de los nutrientes, ayuda a que continúe el desarrollo radicular y se asegure la supervivencia de la 
planta. Ambos nutrientes se hallan relacionados por el efecto negativo que ejerce la carencia de P sobre la fijación atmosférica de N. Por ello, la inoculación con cepas seleccionadas, utilizadas como inoculante, aumenta la eficiencia de la FBN, producción y calidad del cultivo, y en muchos casos el contenido de proteína en el forraje (Perticari, 2006). La fertilización fosfatada es una práctica recomendada al momento de la siembra de alfalfa, ya que muestra respuestas significativas en la producción de forraje cuando la disponibilidad es baja y no está limitada por ningún otro factor (Berg et al., 2005).

El objetivo de este trabajo fue evaluar el efecto de la inoculación con E. meliloti y la fertilización fosfatada sobre la nodulación y productividad de una pastura de alfalfa en el centro de la provincia de Santa Fe.

\section{MATERIALES Y MÉTODOS}

El estudio se realizó en un campo ubicado en la localidad de Esperanza ( $31^{\circ} 24^{\prime} \mathrm{S}, 60^{\circ} 58^{\prime} \mathrm{O}$ ) departamento Las Colonias, provincia de Santa Fe. El clima del área es templado pampeano húmedo. La temperatura media diaria anual es $18^{\circ} \mathrm{C}$ y varía entre $25^{\circ} \mathrm{C}$ en verano y $10^{\circ} \mathrm{C}$ en invierno. El valor medio anual de precipitaciones es $926 \mathrm{~mm}$ y su distribución presenta los menores valores en el período junio-agosto, mientras que los máximos se registran en los meses de diciembre, enero y marzo (Instituto Nacional Tecnología Agropecuaria [INTA], 1991).

El suelo es un Argiudol típico Serie Esperanza con una historia previa de más de cinco años de uso de labranza tradicional y un sistema de rotación de cultivos en base a pasturas de alfalfa. Las características químicas del suelo fueron: $1,89 \%$ de materia orgánica según la metodología propuesta por Walkley y Black (1934); 0,0995 \% de nitrógeno orgánico total según la técnica de Helrich (1990); 7,2 mg/kg de P extractable (Bray y Kurtz, 1945) y pH 6,1 (Ministerio de Agricultura y Ganadería de la provincia de Santa Fe, Argentina [MAG], 1982).

La estimación de la cantidad de rizobios en el suelo se realizó mediante la extracción de diez submuestras en los primeros $0,20 \mathrm{~m}$ de profundidad. El análisis microbiológico se efectuó sobre una muestra compuesta de $10 \mathrm{~g}$ de suelo según la técnica del número más probable (NMP), con diluciones seriadas hasta $10^{-6}$ y cinco repeticiones por dilución (Vincent, 1970).

En abril de 2016 se sembraron 12 kg/ha de semillas viables de alfalfa sin peletear del cultivar Esperanza (GR8), en líneas a 17,5 cm en siembra directa. Se establecieron seis tratamientos divididos en dos grupos: semilla de alfalfa inoculada con E. meliloti y semilla sin inocular. En cada grupo al momento de la siembra se aplicó el fertilizante superfosfato triple (20\% de P) en líneas al costado y por debajo de las semillas utilizando tres dosis: 0 , 20 y $40 \mathrm{~kg} / \mathrm{ha}$ de P. Los tratamientos fueron: testigo sin inocular sin agregado de P (TPO), sin inocular fertilizado con $20 \mathrm{~kg} / \mathrm{ha}$ de P (TP20), sin inocular fertilizado con $40 \mathrm{~kg} / \mathrm{ha}$ de P (TP40), inoculado sin agregado de $\mathrm{P}$ (IP0), inoculado y fertilizado con 20 $\mathrm{kg} / \mathrm{ha}$ de $\mathrm{P}$ (IP20) e inoculado y fertilizado con 40 $\mathrm{kg} / \mathrm{ha}$ de $P$ (IP40). El diseño experimental fue en bloques completamente aleatorizados con cuatro repeticiones y el tamaño de la unidad experimental fue de $80 \mathrm{~m}^{2}$.

Las semillas correspondientes a los tratamientos con inoculación fueron tratadas con un inoculante comercial base turba, cepa B 399 INTA de E. meliloti, dentro de las cuatro horas antes de la siembra. La calidad del inoculante empleado se evaluó según la técnica del NMP en plantas de alfalfa (Vincent, 1970).

A los 45 días de la siembra se cuantificó el número de nódulos formados en raíz principal y secundarias, y se determinó la materia seca (MS) aérea de las plantas. Posteriormente, a los 120, 240 y 480 días de la implantación se evaluaron la biomasa nodular y MS aérea por planta. En todos los muestreos se extrajeron cinco plantas con suelo rizosférico a una profundidad de 0,30 m para cada tratamiento y sus réplicas. Las raíces de las plantas se lavaron con agua, se secaron cuidadosamente con papel absorbente y se separaron los nódulos para su cuantificación o determinación de biomasa nodular. Las plantas se cortaron separando la parte aérea y la raíz por la base del tallo. El material nodular y vegetal fue secado en estufa de aire forzado a $65^{\circ} \mathrm{C}$ hasta peso constante. Además, a los 120, 180 y 240 días posteriores a la siembra se determinaron la MS del forraje $(\mathrm{kg} / \mathrm{ha})$ y la concentración de $\mathrm{N}$ total en la parte aérea de las plantas según el método Kjeldahl (Helrich, 1990). El contenido total de $\mathrm{N}$ en las plantas ( $\mathrm{kg} / \mathrm{ha}$ ) se estimó a partir del producto entre la MS del forraje y la concentración de $\mathrm{N}$. El porcentaje de proteína bruta (PB) se estimó a partir de la concentración de $\mathrm{N}$ total (\%) por el factor 6,25. Los cortes de MS se efectuaron en el estado de $10 \%$ de floración, a una altura de $5 \mathrm{~cm}$ sobre el nivel del suelo, sobre una superficie de $0,25 \mathrm{~m}^{2}$ en cuatro sitios distribuidos al azar por unidad experimental. Luego de recolectar las muestras para determinar la MS del forraje se cortó el resto de las parcelas. 
Los datos meteorológicos registrados durante el período experimental fueron obtenidos de la estación automática agrometeorológica inalámbrica de la Facultad de Ciencias Agrarias, Universidad Nacional del Litoral. En la Figura 1 se presentan la variación de las temperaturas medias mensuales y la distribución de las precipitaciones registradas para el período comprendido entre abril de 2016 y agosto de 2017, y las medias mensuales normales climatológicas (1981-2010) correspondientes a la localidad de Esperanza.

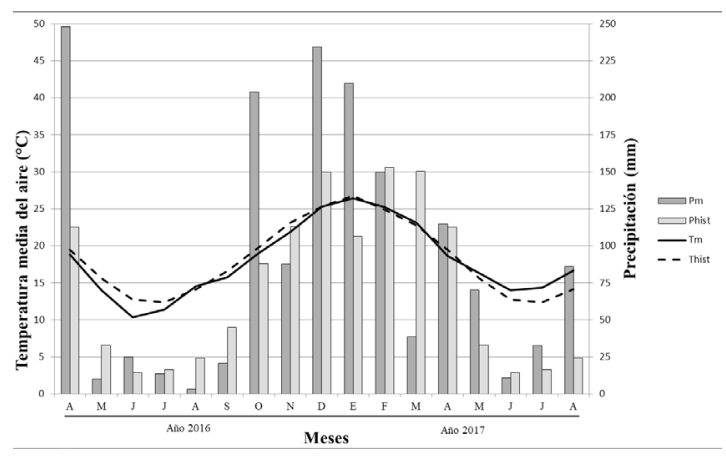

Figura 1. Variación de las temperaturas medias mensuales y distribución de las precipitaciones registradas durante el período de experimentación (abril de 2016-agosto de 2017) y las medias mensuales normales correspondientes a la localidad de Esperanza, Santa Fe (Estación Agrometeorológica de la FCAUNL). Pm: Precipitación mensual, Phist: Precipitación histórica, Tm: temperatura media mensual, Thist: Temperatura media histórica, (1981-2010)

Las temperaturas medias mensuales registradas en los meses de 2016 fueron inferiores a los valores medios históricos, mientras que en los meses de invierno de 2017 presentaron valores más elevados que los normales. La distribución de las precipitaciones fue similar a la distribución histórica reconocida para la región, donde los valores máximos se presentan en los meses de verano. Sin embargo, durante abril, octubre y diciembre de 2016 el volumen de precipitaciones superó al valor normal en 120,132 y $56 \%$ respectivamente, mientras que en mayo y agosto fue 70 y $87 \%$ inferior al valor histórico. En el año 2017, el registro de precipitaciones durante enero, mayo y agosto fue superior a la media histórica, mientras que en el mes de marzo fue $75 \%$ inferior al valor normal de la localidad de Esperanza.

Los resultados fueron sometidos a un análisis de varianza y las medias se compararon según el test de Tuckey $(p<0,05)$. El análisis estadístico se realizó utilizando el software InfoStat (Di Rienzo et al., 2018).

\section{RESULTADOSY DISCUSIÓN}

En el presente trabajo, la población de rizobios del suelo compatibles con alfalfa fue $2.10^{2} \mathrm{NMP} /$ $\mathrm{g}$ de suelo seco. En diversos estudios se ha informado que la nodulación en suelos con bajas poblaciones de rizobios nativos y/o naturalizados dependerá de una correcta inoculación con cepas efectivas. En este sentido, la inoculación resultaría beneficiosa en sitios con una población de rizobios inferior a 100 bacterias por gramo de suelo, independientemente de la eficiencia para fijar $\mathrm{N}$ de los rizobios nativos (Thies, Singleton y Bohlool, 1991; Catroux, Hartmann y Revellin, 2001). La evaluación cuantitativa realizada en el inoculante utilizado en el ensayo mostró una carga bacteriana de 3,4 10 $\mathrm{NMP}$ rizobios/g de inoculante. Este resultado se ajustó a las exigencias del organismo fiscalizador de productos biológicos, coincidiendo con lo informado por Puente, García y Perticari (2011).

El efecto de la inoculación y fertilización con P fue evaluado en distintos momentos de desarrollo de la pastura $(45,120,240$ y 480 días de la siembra) mostrando una interacción significativa para las variables MS de las plantas y biomasa nodular.

A los 45 días de la siembra el $100 \%$ de las plantas de alfalfa presentaron nódulos. El número de nódulos en raíz principal osciló entre 1,38 y 4,94 nódulos por planta y los valores totales entre 1,69 y 13,63 nódulos por planta; mostrando diferencias significativas entre los tratamientos inoculados y con dosis crecientes de $\mathrm{P}$, sin registrarse interacción entre la inoculación y la aplicación de $P$ (Tabla 1). A medida que se incrementó la dosis de $\mathrm{P}$ aumentó el número de nódulos por planta tanto en los tratamientos inoculados como en los sin inoculación, siendo significativo el efecto de la inoculación sobre el número de nódulos de la raíz principal. Este comportamiento sería atribuido a un mejor ambiente nutricional, estimulante de la infección y desarrollo inicial de los rizobios (DíazZorita, Grosso, Fernández-Canigia y Duarte, 1999).

En este estadio, la MS de las plantas del tratamiento TPO mostró el valor más bajo en relación a los tratamientos inoculados y con dosis crecientes de $\mathrm{P}$ siendo las diferencias significativas (Tabla 2). Existe consenso respecto a que poblaciones menores a 100 rizobios/g de suelo pueden producir una baja acumulación de MS en plantas de alfalfa debido a una nodulación insuficiente (Brockwell, Holliday y Pilka, 1988). Estudios realizados en diferentes asociaciones rizobio-leguminosa concluyeron que sería poco probable que bajas poblaciones de rizobios 
Tabla 1. Número de nódulos de plantas de alfalfa a los 45 días de la siembra. Respuesta a la aplicación de tasas crecientes de fósforo y la inoculación con rizobios

\begin{tabular}{lcc}
\hline Tratamiento $^{1}$ & $\begin{array}{c}\text { Nódulos RP } \\
\left(\mathrm{n}^{\circ} / \text { planta }\right)\end{array}$ & $\begin{array}{c}\text { Nódulos RS } \\
\left(\mathrm{n}^{\circ} / \text { planta }\right)\end{array}$ \\
\hline TP0 & $1,38 \pm 1,36 \mathrm{a}$ & $0,31 \pm 0,70 \mathrm{a}$ \\
TP20 & $3,38 \pm 1,82 \mathrm{bc}$ & $4,25 \pm 1,73 \mathrm{~b}$ \\
TP40 & $3,31 \pm 1,30 \mathrm{bc}$ & $6,94 \pm 2,82 \mathrm{~cd}$ \\
P & $<0,0001$ & $<0,0001$ \\
IP0 & $2,19 \pm 1,60 \mathrm{ab}$ & $0,69 \pm 1,54 \mathrm{a}$ \\
IP20 & $4,81 \pm 0,83 \mathrm{~cd}$ & $4,88 \pm 3,28 \mathrm{bc}$ \\
IP40 & $4,94 \pm 1,65 \mathrm{~d}$ & $8,69 \pm 3,63 \mathrm{~d}$ \\
I & $<0,0009$ & $<0,1483$ \\
I*P & $<0,6842$ & $<0,7665$ \\
\hline
\end{tabular}

1:TP0: Testigo sin inocular sin agregado de P; TP20: sin inocular fertilizado con $20 \mathrm{~kg} / \mathrm{ha}$ de P; TP40: sin inocular fertilizado con 40 kg/ha de P; IP0: inoculado E. meliloti sin agregado de P; IP20: inoculado y fertilizado con $20 \mathrm{~kg} /$ hade P; IP40: inoculado y fertilizado con $40 \mathrm{~kg} /$ ha de P; I: inoculado; P: fósforo. 2: RP: Raíz Principal. 3: RS: Raíces Secundarias. Letras distintas en las columnas indican diferencias significativas $(p \leq 0,05)$

en el suelo fueran suficientes para inducir una nodulación adecuada en la planta huésped (Brockwell, Bottomley y Thies, 1995; Howieson y Ballard, 2004). La inoculación en el tratamiento (IPO) incrementó el $47 \%$ la MS aérea de las plantas respecto al TPO. La aplicación de dosis crecientes de $\mathrm{P}$ aumentó significativamente la MS de las plantas conduciendo a una producción de 80,93 $\mathrm{mg} /$ planta en el testigo y $92,90 \mathrm{mg} / \mathrm{planta}$ en el tratamiento inoculado para un nivel de $40 \mathrm{~kg}$ de P/ha.

Asimismo, hubo una asociación positiva entre la MS aérea de las plantas de alfalfa y la cantidad de nódulos a los 45 días de la siembra (Figura 2A) y la biomasa nodular a los 120 días (Figura 2B), sugiriendo la importancia del proceso de nodulación y de FBN sobre la producción y acumulación de biomasa del cultivo (Gan, Stulen, Van Keulen y Kuiper, 2003).

Tabla 2. Materia seca de plantas de alfalfa a los 45 días de la siembra. Respuesta a la aplicación de tasas crecientes de fósforo y la inoculación con rizobios

\begin{tabular}{lcccc}
\hline Tratamiento $^{1}$ & \multicolumn{2}{c}{$\mathrm{T}^{2}$} & $\mathrm{I}^{3}$ & । \\
& MS (mg/planta) & MS (mg/planta) & \\
\hline P0 & $18,04 \pm 4,3 \mathrm{a}$ & $26,45 \pm 5,86 \mathrm{~b}$ & $<0,0001$ \\
P20 & $51,23 \pm 17,7 \mathrm{c}$ & $84,88 \pm 3,06 \mathrm{~d}$ & $<0,0001$ \\
P40 & $80,93 \pm 3,15 \mathrm{~d}$ & $92,90 \pm 5,63 \mathrm{e}$ & $<0,0001$ \\
P & $<0,0001$ & $<0,0001$ & $<0,0001$ \\
\hline 1:P0:
\end{tabular}

1:P0: sin agregado de P; P20: fertilizado con $20 \mathrm{~kg} / \mathrm{ha}$ de $\mathrm{P} ; \mathbf{P} \mathbf{4 0}$ : fertilizado con $40 \mathrm{~kg} / \mathrm{ha}$ de P. 2: T: Testigo. 3: I: Inoculado E. meliloti. I: inoculado. P: fósforo. Letras distintas en las columnas indican diferencias significativas $(p \leq 0,05)$.
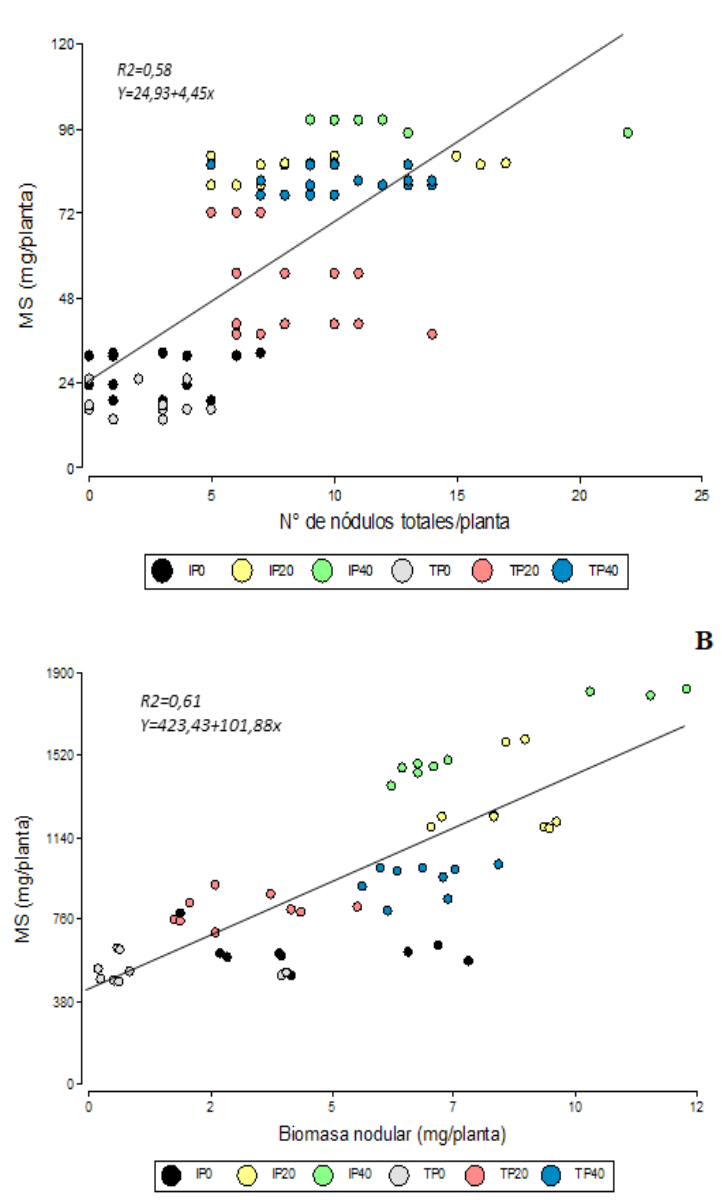

Figura 2. A: Relación entre el número de nódulos y la materia seca de las plantas a los 45 días de la siembra. B: Relación entre la biomasa seca de los nódulos y la materia seca de las plantas a los 120 días de la siembra. Análisis de las plantas provenientes de los tratamientos testigos e inoculados con dosis crecientes de aplicación de fertilizante fosfatado. MS: Materia seca ( $N=120)$

A los 120 días de la siembra, la fertilización con $\mathrm{P}$ incrementó la biomasa de los nódulos y la MS aérea de las plantas según se muestra en la Figura $3 \mathrm{~A}$, presentando interacción entre la inoculación y la aplicación de fertilizante fosfatado $(p<0,0001)$. Sulieman, Ha, Schulze y Tran (2013) hallaron aumentos en el número y en la biomasa de los nódulos de Medicago truncatula al incrementar los aportes de P. Estudios realizados en el cultivo de soja mostraron resultados similares (Fontanetto, Díaz-Zorita y Vivas, 2004; Miao, Qiao, Han y An, 2007), sugiriendo que el $P$ sería limitante para la formación y crecimiento de los nódulos. Con relación a la MS aérea de las plantas la inoculación aumentó un 60 \% para IP20 y $67 \%$ para IP40 en relación a los tratamientos sin inocular con las 
mismas dosis de $\mathrm{P}$.

En la Figura 3B se muestra que la fertilización fosfatada produjo un incremento $(p<0,05)$ de la MS aérea de las plantas a los 240 días de la siembra,

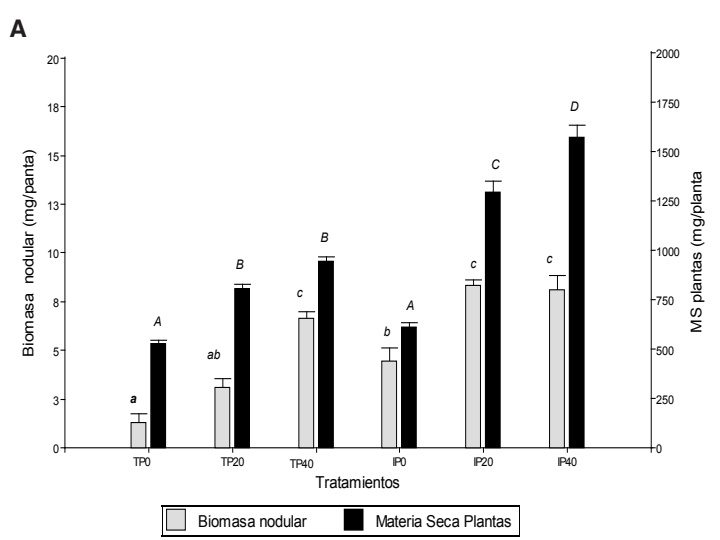

B

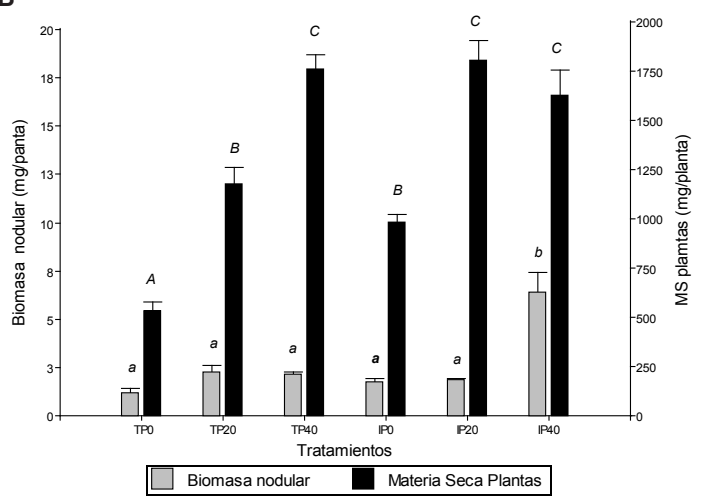

C

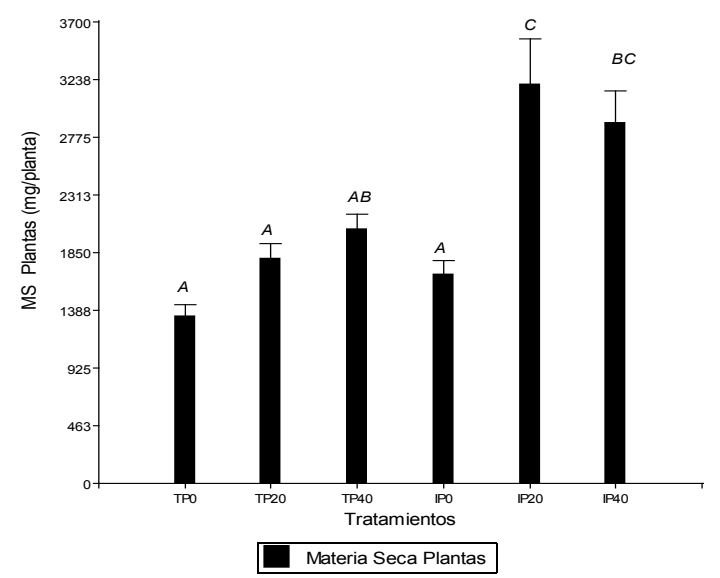

Figura 3. Efecto de la inoculación y fertilización con fósforo sobre el peso seco y la biomasa nodular de las plantas de alfalfa. A: Materia seca de las plantas y biomasa nodular de los nódulos a los 120 días de la siembra. B: Materia seca de las plantas y biomasa nodular de los nódulos a los 240 días de la siembra. C: Materia seca de las plantas a los 480 días de la siembra. Letras distintas en las columnas indican diferencias significativas $(p \leq 0,05)$ presentando interacción entre la inoculación y la fertilización $(p<0,0001)$. En los tratamientos IP0 e IP20 la MS fue un 84 y $53 \%$ superior respectivamente, en relación a los tratamientos sin inocular con las mismas dosis de P aplicadas. Sin embargo, la biomasa de los nódulos fue significativamente mayor solo cuando las plantas de alfalfa fueron inoculadas y fertilizadas con el nivel máximo de $P$.

A los 480 días de la siembra las raíces de las plantas de alfalfa no presentaron nódulos, según se observa en la Figura 3C. A pesar de ello, la fertilización fosfatada indujo cambios significativos en la MS de las plantas inoculadas, mostrando interacción entre la inoculación y la aplicación de $P(p<0,05)$. Según Racca y González (2007), a medida que avanza el ciclo del cultivo sería frecuente observar las raíces de las plantas de alfalfa sin nódulos en los primeros $30040 \mathrm{~cm}$ del perfil del suelo. El sistema radical crecería buscando mayor profundidad guiado por la disponibilidad de agua y emitiendo raicillas capaces de aprovechar el contenido de agua subsuperficial. Estas raicillas receptivas a las cepas de rizobios naturalizadas o provenientes del inoculante aplicado formarían nuevos nódulos, que favorecerían el incremento de la MS de las plantas.

Los niveles de producción de MS de alfalfa en el año de implantación, independientemente del tratamiento considerado, fueron menores a los obtenidos con siembras otoñales en trabajos similares en el centro de la provincia de Santa Fe (Fontanetto y Bianchini, 2007; Vázquez et al., 2010), por lo que se estimó que las condiciones meteorológicas registradas durante el período experimental, el bajo contenido de materia orgánica y la escasa dotación de nutrientes del suelo pudieron haber afectado la producción de las pasturas. Además, la frecuencia de cosecha del forraje pudo haber ocasionado una subestimación en la determinación de la acumulación de forraje. Períodos prolongados de rebrote entre cosechas pudieron haber ocasionado pérdidas por senescencia del forraje producido. Por otro lado, Berardo, Marino y Erht (2007) encontraron una reducción en la producción del primer año del cultivo de alfalfa debido a la escasez de precipitaciones registradas durante la implantación.

La aplicación de $\mathrm{P}$ incrementó significativamente $(p<0,05)$ la producción de MS, obteniéndose para los tratamientos testigo (TP0) y para la máxima dosis de P utilizado (TP40) 227 y $431 \mathrm{~kg} / \mathrm{ha}$ de MS a los 120 días, 427 y $1193 \mathrm{~kg} / \mathrm{ha}$ de MS a los 180 días, y 677 y $835 \mathrm{~kg} / \mathrm{ha}$ de MS a los 240 días, según se muestra en la Figura $4 \mathrm{~A}$. La técnica de la inoculación provocó un incremento significativo 
de la producción de MS que osciló entre 23 y $58 \%$ para los tratamientos con el máximo nivel de fertilizante aplicado en los tres momentos de muestreo.

En la Figura 4B se presenta el contenido de $\mathrm{N}$ en la MS de las plantas de alfalfa en los tratamientos evaluados para los tres momentos de muestreo. La inoculación aumentó el $\mathrm{N}$ acumulado en la MS del forraje con la aplicación de dosis crecientes de $\mathrm{P}$, manteniendo la misma tendencia para la producción de MS. El uso de inoculante con cepas de rizobios seleccionadas incrementó el $28 \%$

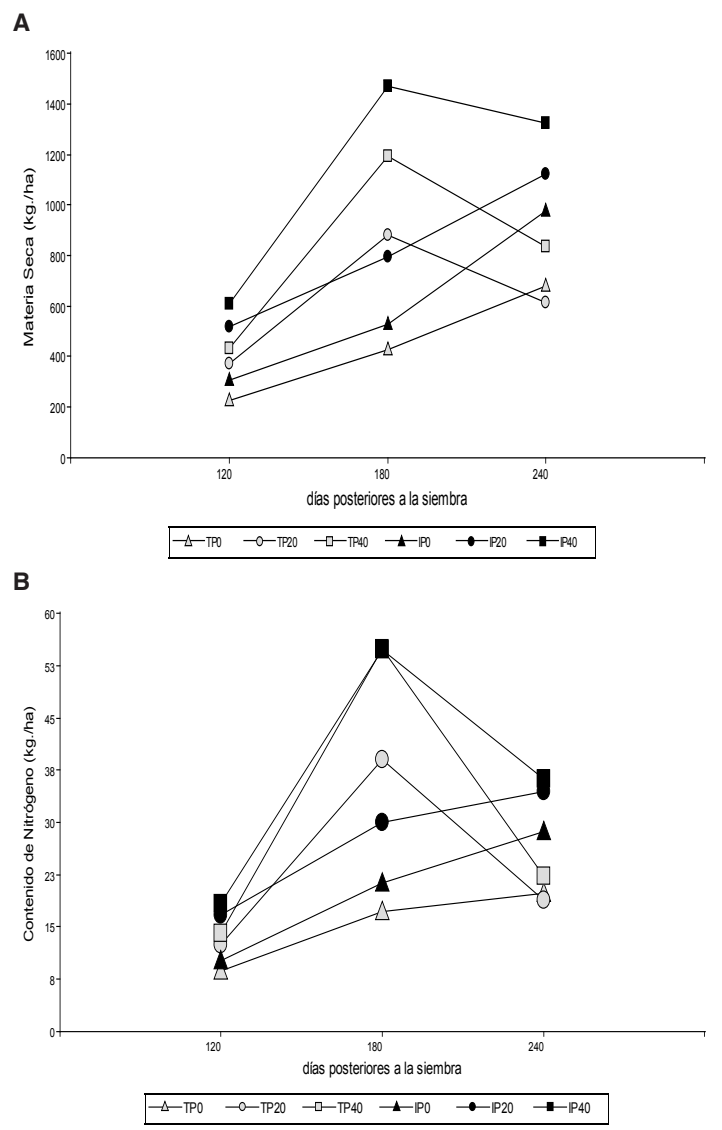

Figura 4. Efecto de la inoculación y aplicación de dosis crecientes de fósforo sobre la producción de alfalfa. A. Producción de materia seca. B. Contenido de nitrógeno en la materia seca

y $59 \%$ el contenido de $\mathrm{N}$ en la MS a los 120 y 240 días de la siembra respectivamente, en los tratamientos con el agregado de $40 \mathrm{~kg} / \mathrm{ha}$ de $\mathrm{P}$.

El contenido estimado de PB de las plantas de alfalfa varió entre $24,4 \%$ a los 120 días y $16,9 \%$ a los 240 días de la siembra del cultivo, evidenciando el estado avanzado de acumulación de biomasa aérea debido a los períodos prolongados de cosecha. Sin embargo, estos valores de PB concuerdan con los obtenidos por Basigalup, Castell y Giaveno (2004) y Plevich et al., (2012). En este aspecto, Nescier y Dalla Fontana (2003), en un ensayo realizado en la localidad de Esperanza, Santa Fe, sobre un cultivo de alfalfa fertilizado con $\mathrm{P}$, con y sin inoculación de la semilla, no encontraron cambios significativos en la PB de las plantas en los dos primeros cortes durante el año de implantación. Sin embargo, Dalla Fontana, Nescier, Pennisi, Longoni y Contini (2007) en la región central de la cuenca lechera santafesina, observaron que en el verano la PB del forraje de alfalfa fertilizado con P y azufre aumentó significativamente en relación a las plantas sin fertilizar. Estos autores sugirieron que la aplicación de $P$ en suelos con deficiencia de este nutriente promovería la formación de nódulos y la FBN para la síntesis de proteína en pasturas de alfalfa.

\section{CONCLUSIONES}

En las condiciones del presente estudio, la inoculación con E. meliloti y la aplicación de dosis crecientes de fertilizante fosfatado incrementaron la producción del cultivo de alfalfa.

La técnica de la inoculación mostró un aumento de la producción de materia seca entre 23 y $58 \%$ para los tratamientos con el máximo nivel de fertilizante a los 120, 180 y 240 días de la siembra.

La aplicación de dosis crecientes de fertilizante fosfatado produjo un aumento en el número de nódulos, biomasa nodular y MS de las plantas. Este comportamiento estaría asociado con mejoras en el ambiente nutricional logrando un mayor rendimiento del cultivo.

\section{BIBLIOGRAFÍA}

Anglade, J., Billen, G. y Garnier, J. (2015). Relationships for estimating $\mathrm{N} 2$ fixation in legumes: incidence for $\mathrm{N}$ balance of legume-based cropping systems in Europe. Ecosphere, 6 (3), 1-24. DOI: https://doi.org/10.1890/ ES14-00353.1

Basigalup, D. H., Castell, C. V. y Giaveno, C. D. (2003). Response to selection for lower initial rate of dry matter disappearance in the development of a bloat-tolerant non-dormant alfalfa population. Journal of Genetics and Breeding, 57, 31-37.

Basigalup, D. H., Rossanigo, R. y Ballario, M. (2007). Capítulo 1. Panorama actual de la alfalfa en la Argentina. En Basigalup, D. H. (Ed.), El cultivo de la alfalfa en la Argentina (15-25). Buenos Aires, 
Argentina: Ediciones INTA. Recuperado de: https:// inta.gob.ar/sites/default/files/inta-el_cultivo_de_la_ alfalfa_en_la_argentina.pdf

Berardo, A. y Marino M. A. (2001). Producción de forraje de alfalfa bajo diferentes niveles de nutrición fosfatada en el sudeste bonaerense. Revista Argentina de Producción Animal, 20 (2), 93-101.Recuperado de: http://laboratoriofertilab.com.ar/Trabajos/Alfalfa3.pdf

Berardo, A., Marino, M. A. y Erht, S. (2007). Producción de forraje de alfalfa con aplicación de fósforo superficial y profunda. RIA. Revista de Investigaciones Agropecuarias, 36 (1), 97-113. Recuperado de: http:// www.redalyc.org/articulo. $.0 a ?$ id $=86436106$

Berg, W. K., Cunningham, S. M., Brouder, S. M., Joern, B. C., Johnson, K. D., Santini, J. y Volenec, J. J. (2005). Influence of phosphorus and potassium on alfalfa yield and yield components. Crop Science, 45, 297-304. DOI: https://doi.org/10.2135/cropsci2005.0297

Bray, R. H. y Kurtz, L. (1945). Determination of total, organic and available forms of phosphorus in soils. Soil Science, 59 , 39-45.

Brockwell J., Holliday R. A. y Pilka A. (1988). Evaluation of the symbiotic nitrogen-fixing potential of soils by direct microbiological means. Plant and Soil, 108, 163-170. DOI: https://doi.org/10.1007/BF02370111

Brockwell, J., Bottomley, P. J. y Thies, J. E. (1995). Manipulation of rhizobia microflora for improving legume productivity and soil fertility: a critical assessment. Plant and Soil, 174, 143-180. DOI: https:// doi.org/10.1007/BF00032245

Campillo, R., Urquiaga, S., Pino, I. y Montenegro, A. (2003). Estimación de la fijación biológica de nitrógeno mediante la metodología de 15N. Agricultura Técnica, 63 (2), 169-179. DOI:

http://dx.doi.org/10.4067/S0365-28072003000200006

Catroux, G., Hartmann, A. y Revellin, C. (2001). Trends in rhizobial inoculant production and use. Plant and Soil, 230, 21-30. DOI: https://doi. org/10.1023/A: 1004777115628

Dalla Fontana, L. A., Nescier, I. M., Pennisi, D. T., Longoni, M. L. y Contini, L. (2007). Efecto de la fertilización sobre la calidad de plantas de alfalfa (Medicago sativa L.) a través del ciclo de producción. Revista FAVE - Ciencias Veterinarias, 6, 87-93. Recuperado de: https://bibliotecavirtual.unl.edu.ar/publicaciones/ index.php/FAVEveterinaria/article/view/1445/2309

Darwich, N. A. (1983). Niveles de $P$ disponible en suelos pampeanos. (Informativo de investigaciones agrícolas $N^{\circ}$ 409-412, 1-5). Buenos Aires, Argentina: Instituto Nacional de Tecnología Agropecuaria.

Di Rienzo, J. A., Casanoves, F., Balzarini, M. G., Gonzalez, L., Tablada, M. y Robledo, C. W. (2018). Infostat (versión 2018) [Software] Córdoba, Argentina: Grupo
InfoStat, FCA, Universidad Nacional de Córdoba. URL: http://www.infostat.com.ar

Díaz-Zorita, M., Grosso, G., Fernández-Canigia, M. V. y Duarte, G. (1999). Efectos de la ubicación de un fertilizante nitrógeno-fosfatado sobre la nodulación y la producción de soja en siembra directa en la región de la pampa arenosa, Argentina. Ciencia del Suelo, 17 (2), 62. Recuperado de: https://www.suelos. org.ar/publicaciones/vol_17n2/diaz-zorita_62-65.pdf

Díaz Zorita, M y Gambaudo, S. (2007). Capítulo 11. Fertilización y encalado en alfalfa. En Basigalup, D. H. (Ed.). El cultivo de la alfalfa en la Argentina (227-246). Buenos Aires, Argentina: Ediciones INTA. Recuperado de: https://inta.gob.ar/sites/default/files/ inta-el_cultivo_de_la_alfalfa_en_la_argentina.pdf

Fontanetto, H., Díaz-Zorita, M. y Vivas, H. (2004). Inoculación y fertilización con fósforo y azufre sobre la nodulación y los rendimientos de soja. Publicación Miscelánea, 102, 46-52. Recuperado de: http:// rafaela.inta.gov.ar/publicaciones/documentos/ miscelaneas/102/misc102_p46.pdf

Fontanetto, H. y Bianchini, A. (2007). Fertilización fosfatada y azufrada de alfalfa a la siembra y al año de implantación en el centro-este de Santa Fe. Informaciones Agronómicas del Cono Sur, 36, 22-25. Recuperado de: http://www.forratec.com.ar/manuales/ pdfs/28-20140821142253-pdfEs.pdf

Gan, Y., Stulen, I., Van Keulen, H. y Kuiper, P. J. C. (2003). Effect of $N$ fertilizer top-dressing at various reproductive stages on growth, $\mathrm{N}_{2}$ fixation and yield of three soybean (Glycine max (L.) Merr.) genotypes. Field Crops Research, 80 (2), 147-155. DOI: https:// doi.org/10.1016/S0378-4290(02)00171-5

Helrich, K. (1990). Official methods of analysis of the Association of Official Analytical Chemists. (15a ed.). Association of Official Analytical Chemists Inc. Arlington, Virginia, Estados Unidos: Association of Official Analytical Chemists Inc.

Howarth, R. E. (1988). Antiquality Factors and Nonnutritive Chemical Components. En: Hanson, A. A., Barnes, D. K. y Hill, R. R. Jr. (Eds), Alfalfa and Alfalfa Improvement: Vol. 29Agronomy Monographs (493-514). Madison, Winsconsin, EE. UU.: American Society of Agronomy, Inc., Crop Science Society of America, Inc., Soil Science Society of America, Inc. DOI: https://doi.org/10.2134/agronmonogr29.c15

Howieson, J. G. y Ballard, R. (2004). Optimising the legume symbiosis in stressful and competitive environments within southern Australia - some contemporary thoughts. Soil Biology and Biochemistry, 36 (8), 1261-1273. DOI: https://doi.org/10.1016/j. soilbio.2004.04.008

IInstituto Nacional Tecnología Agropecuaria, Estación Experimental Agropecuaria Rafaela. (INTA, EEA 
Rafaela) (1991) Carta de suelos de la República Argentina. Esperanza - Pilar, Santa Fe. Argentina: Ediciones INTA.

Jensen, E. S. y Hauggaard-Nielsen, H. (2003). How can increased use of biological N2 fixation in agriculture benefit the environment? Plant and Soil, 252, 177-186. DOI: https://doi.org/10.1023/A:1024189029226

Miao, S. J., Qiao, Y. F., Han, X. Z. y An, M. (2007). Nodule formation and development in soybeans (Glycine max L.) in response to phosphorus supply in solution culture. Pedosphere, 17 , 36-43. DOI: https://doi. org/10.1016/S1002-0160(07)60005-8

Ministerio de Agricultura y Ganadería de la provincia de Santa Fe (M.A.G.) (1982). Dirección General de Extensión e Investigaciones Agropecuarias. Toma de muestras y Determinaciones Analíticas en Suelos y Aguas. Santa Fe. Argentina: M.A.G. Santa Fe.

Nescier, I. M. y Dalla Fontana, L. A. (2003). Inoculación y fertilización fosfatada sobre el contenido proteico de alfalfa. Revista FAVE - Ciencias Agrarias, 2, 6770. Recuperado de https://bibliotecavirtual.unl.edu. ar/publicaciones/index.php/FAVEAgrarias/article/ view/77/98

Perticari, A. (2006). Pasturas de alfalfa: importancia de una adecuada inoculación. Presentado en el II Congreso Nacional de Conservación y Uso de Forrajes. INTA Castelar.Buenos Aires, Argentina. Recuperado de: https://docplayer.es/114985916-Pasturas-de-alfalfaimportancia-de-una-adecuada-inoculacion.html

Plevich, J. O., Delgado, A. R., Saroff, C., Tarico, J. C., Crespi, R. J. y Barotto, O. M (2012). El cultivo de alfalfa utilizando agua de perforación, agua residual urbana y precipitaciones. Revista Brasileira de Engenharia Agrícola e Ambiental, 16 (12), 1353-135 DOI: http:// dx.doi.org/10.1590/S1415-43662012001200013

Puente, M., García, J. y Perticari, A. (2011). Inoculación de leguminosas forrajeras con rizobios eficientes. Documento presentado en el IV Congreso de Producción y Uso de Forrajes. Buenos Aires, Argentina: Producir XXI y Estudio Ganadero Pergamino. Recuperado de http://www.produccionanimal.com.ar/produccion_y_manejo_pasturas/ pasturas_cultivadas_alfalfa/120-inoculacion.pdf

Quiñonez, A., Romero, L., Dalla Fontana, L. A., Longoni, M. y Colombo, S. (2008). Fertilización fosforada y azufrada en alfalfa. Revista FAVE-Ciencias Agrarias, 7, 75-80. Recuperado de: https://bibliotecavirtual.unl. edu.ar/publicaciones/index.php/FAVEAgrarias/article/ view/1330/2098

Racca, R., Collino, D., Dardanelli, J., Basigalup, D., González, N., Brenzoni, E.,... y Balzarini, M. (2001). Contribución de la fijación biológica de nitrógeno a la nutrición nitrogenada de la alfalfa en la región pampeana. Ediciones INTA. Buenos Aires, Argentina:
Ediciones INTA. Recuperado de: https://forratec.com. ar/uploads/191-20180215162608-pdfEs.pdf

Racca, R. y González, N. (2007). Capítulo 4. Nutrición nitrogenada de la alfalfa e impacto de la fijación biológica del nitrógeno. En: D. H. Basigalup (Ed.), El cultivo de la alfalfa en la Argentina (67-79). Buenos Aires, Argentina: Ediciones INTA. Recuperado de: https://inta.gob.ar/sites/default/files/inta-el_cultivo_ de_la_alfalfa_en_la_argentina.pdf

Ribet, J. y Drevon, J. J. (1995). Increase in permeability to oxygen and in oxygen uptake of soybean nodules under limiting phosphorus nutrition. Physiolgia Plantarum, 94 (2), 298-304. DOl: https://doi. org/10.1111/j.1399-3054.1995.tb05315.x

Sainz Rozas, H. R., Echeverría, H. E. y Angelini, H. P. (2012). Fósforo disponible en suelos agrícolas de la región Pampeana y Extrapampeana argentina. Revista de Investigaciones Agropecuarias (RIA), 38, 33-39. Recuperado de: http://www.redalyc.org/ articulo.oa?id=86423614007

Sevilla, G. y Agnusdei, M. G. (2016). Efecto del agregado de fósforo y nitrógeno en el crecimiento de cultivos de alfalfa en rebrotes de primavera y verano en un suelo vertisol de Entre Ríos. Revista de Investigaciones Agropecuarias (RIA), 42 (1), 93-101. Recuperado de: http://www.redalyc.org/articulo.oa?id=86445998017

Silva, C., Kan, F. L. y Martínez-Romero, E. (2007). Population genetic structure of Sinorhizobium meliloti and S. medicae isolated from nodules of Medicago spp. in Mexico. FEMS Microbiology Ecology, 60 (3), 477-489. DOI: https://doi.org/10.1111/j.15746941.2007.00301.x

Sulieman, S., Ha, C. V., Schulze, J. y Tran, L. S. P. (2013). Growth and nodulation of symbiotic Medicago truncatula at different levels of phosphorus availability. Journal of Experimental Botany, 64 (10), 2701-2712. DOI: https://doi.org/10.1093/jxb/ert122

Tejerizo, G. T., Rogel, M. A., Ormeño-Orrillo, E., Althabegoiti, M. J., Nilsson, J. F., Niehaus, K.,...y Pistorio, M. (2016). Rhizobium favelukesii sp. nov., isolated from the root nodules of alfalfa (Medicago sativa L). International Journal of Systematic and Evolutionary Microbiology, 66(11), 4451-4457. DOI: https://doi.org/10.1099/ijsem.0.001373

Thies, J. E., Singleton, P. W. y Bohlool, B. B. (1991). Influence of the size of indigenous rhizobial populations on establishment and symbiotic performance of introduced Rhizobia on field-grown legumes. Applied and environmental microbiology, 57, 19-28. DOI: https://doi.org/10.1128/aem.57.1.19-28.1991

Vázquez, M., Terminiello, A., Casciani, A., Millán, G., Gelati, P., Guilino, F... y García, M. (2010). Influencia del agregado de enmiendas básicas sobre la producción de alfalfa (Medicago sativa L.) en ámbitos 
templados argentinos. Ciencia del suelo, 28 (2),141154. Recuperado de: http://www.suelos.org.ar/ publicaciones/vol_28n2/28(2)\%20141-154.pdf

Vincent, J. M. (1970). A Manual for the Practical Study of the Root-Nodule Bacteria: IBP Handbook № 15 International Biological Programme. Oxford, Reino Unido: Blackwell Scientific.

Vivas, H. S. y Guaita, M. S. (1997). Respuesta a la fertilización fosfatada de alfalfa en un año caracterizado por estrés hídrico. Publicación Miscelánea Nº 84. EEA INTA Rafaela, Santa Fe. Recuperado de: http:// rafaela.inta.gov.ar/info/miscelaneas/inta_rafaela_ miscelanea_084.pdf

Walkley, A. y Black, I. A. (1934). An examination of the dagthareff method for determining soil organic matter and proposed modification of the chromic acid titration method. Soil Science, 37 (1), 27-38 DOI: https://doi. org/10.1097/00010694-193401000-00003 